AperTO - Archivio Istituzionale Open Access dell'Università di Torino

\title{
Performance results of the trigger logic implemented in EUSO-SPB
}

\section{This is a pre print version of the following article:}

Original Citation:

Availability:

This version is available http://hdl.handle.net/2318/1730088

since 2020-02-23T17:52:14Z

Published version:

DOI:10.1016/j.nima.2018.11.002

Terms of use:

Open Access

Anyone can freely access the full text of works made available as "Open Access". Works made available under a Creative Commons license can be used according to the terms and conditions of said license. Use of all other works requires consent of the right holder (author or publisher) if not exempted from copyright protection by the applicable law. 


\title{
NIMA POST-PROCESS BANNER TO BE REMOVED AFTER FINAL ACCEPTANCE
}

\section{Performance results of the trigger logic implemented in EUSO-SPB}

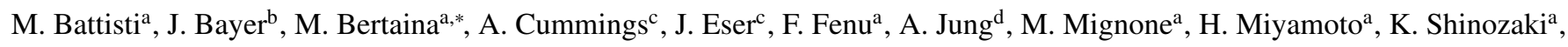
for the JEM-EUSO Collaboration

\author{
${ }^{a}$ Department of Physics, University of Torino $\mathcal{E}$ INFN Torino, Torino, Italy \\ ${ }^{b}$ University of Tübingen, Kepler Center for Astro and Particle Physics, Tübingen, Germany \\ ${ }^{c}$ Department of Physics, Colorado School of Mines, Mines, US \\ ${ }^{d} A P C$, Univ Paris Diderot, CNRS/IN2P3, France
}

\begin{abstract}
EUSO-SPB (Extreme Universe Space Observatory - Super Pressure Balloon) [1] is the first path-finder mission of the JEM-EUSO program which hosted a trigger logic to detect Ultra-High Energy Cosmic Rays (UHECRs) following the concept envisaged for the main mission. The performance of the implemented logic was verified by means of dedicated tests carried out in a field campaign at the Telescope Array (TA) site where the EUSO-SPB detector was exposed to the night sky luminosity and to a variety of point-like light sources such as stars, meteors, and laser shots to mimic UHECR tracks. Finally, the logic was tested on board the EUSO-SPB flight. Results satisfied the requirements conceived for the First Level Trigger (FLT) envisaged for JEM-EUSO as described in [2].
\end{abstract}

Keywords: Front End, Trigger, DAQ and Data Management, JEM-EUSO

PACS: 29.85.Ca, 96.50.sd

\section{Introduction and trigger logic}

EUSO-SPB launched from Wanaka New Zealand on April 25th 2017 on a NASA Super Pressure Balloon and flew more than 12 days. During the flight EUSO-SPB collected $\sim 100 \mathrm{k}$ events. Most of them were successfully downloaded on ground and processed. EUSO-SPB mounted on-board an autonomous FLT similar to the one foreseen for JEM-EUSO [2]. The Focal Surface (FS) of EUSO-SPB was based on MAPMTs (Hamamatsu Photonics R11265-03-M64) which have $8 \times 8$ pixels and for readout purposes $2 \times 2$ MAPMTs are grouped into one Elementary Cell (EC). The FLT works at the level of these ECs. Nine ECs form one PDM [3].

The FLT [2] rejects most of the fluctuations of the UV nightglow as well as electronic noise by requiring a locally persistent signal that lasts a few Gate Time Units (GTUs, 1 GTU $=2.5 \mu \mathrm{s}$ ) above the average background. In the FLT pixels are grouped in cells of $3 \times 3$ pixels. When a pixel in a cell detects a number of counts equal, or higher than, a preset threshold $N$, the FLT logic checks if for a certain number of following GTUs $(R)$ in a slot of

\footnotetext{
${ }^{*}$ Corresponding authors

Email address: bertaina@to.infn.it (M. Bertaina)
}

consecutive GTUs $(P)$, there is at least one pixel in the same cell with an activity equal to, or higher than $N$, and the total number of photo-electrons integrated in the cell is higher than a preset value $S$. If this condition is verified a pre-trigger is issued. In case the pre-trigger activation persists for less than 73 GTUs the event is finally triggered. If the persistence lasts longer the event is considered not to be compliant with an UHECR duration and the trigger is not issued. $R$ and $P$ define the trigger mode suitable for the JEM-EUSO path-finder. In case of EUSO-TA [4] or EUSO-SPB where the location of the Extensive Air Shower (EAS) can vary between 1 to $30 \mathrm{~km}, P$ values such as 0 or 1 are the most appropriate; consequently, $R$ can be 0 or 1 . On the other hand, for JEM-EUSO, looking at $400 \mathrm{~km}$ distant EAS, $P=4$ and $R=2$. Having fixed $P$ and $R$, the $N$ and $S$ parameters are set as a function of the average background level on each MAPMT independently to keep the rate of triggers on false positives at $\sim 1 \mathrm{~Hz} / \mathrm{EC}$. The parameters can also be set via an external command. For each pixel an average background level is calculated every 128 GTUs ( $320 \mu$ s). The pixel with the highest average count determines the thresholds for the entire MAPMT. This method takes more precisely into account the non-uniformity among pixels, as well as the presence of man-made sources with very limited spatial extension. All the 
artificial sources such as cities, ships can be considered static at this level. The FLT and all the control logic and interfaces was implemented on a Xilinx Virtex 6 model XC6VLX360T FPGA and it used around $34 \%-43 \%$ of the available resources.

\section{Trigger tests during the Utah campaign}

In October 2016, the fully assembled EUSO-SPB detector was tested for a week at the EUSO-TA site to measure the overall response of the detector and to calibrate it by means of the Central Laser Facility (CLF) of TA and a portable Ground Laser System (GLS) of Colorado School of Mines. This time the FLT trigger was tested on the entire PDM for about a week. The trigger logic was set with $P=1$ and $R=1$. In normal sky conditions, the FLT rate was below $1 \mathrm{~Hz}$ on the entire PDM, increasing to few $\mathrm{Hz}$ during thunderstorms outside the FoV (the system could not have been operated with thunderstorms in the FoV) or during the passage of airplanes. This was due to the fact that the strobe lights of airplanes have very sharp raising phase of the signal, with longer time decay. Being just above threshold, they failed to be recognized as slow events. The fact that in normal conditions stars were not creating huge bursts of triggers proved that the logic is capable of dealing with very slow but bright moving objects. Only rarely, when a bright star was passing through different MAPMTs and the light was scattered in an irregular way from the filters, a slight increase in the trigger rate was observed. Similar situation occurred in presence of meteoroids. A few of them triggered EUSO-SPB but at the level of no more than $10 \mathrm{~Hz}$, which means that the logic successfully avoided too high trigger rates. Most of the time, EUSO-SPB trigger logic was tested using CLF and GLS laser shots. The GLS laser is a mobile system which was set at a distance of 25 $\mathrm{km}$ from EUSO-SPB and EUSO-TA with a laser intensity in the range of 1 to $86 \mathrm{~mJ}$. As the laser itself is steerable, the geometry of the laser track can be varied more freely, mimicking EAS of different energy and direction. The GLS turned to be essential to provide a first estimation of the energy threshold of the FLT to trigger on EAS-like events. By comparing the laser signal on the FS and the expectation from simulations of EAS, the result was that laser intensity giving $\sim 50 \%$ trigger efficiency is equivalent to that expected from $\sim 3 \times 10^{18} \mathrm{eV}$ and $45^{\circ}$ inclined EAS as seen from EUSO-SPB at an altitude of $30 \mathrm{~km}$. This was a positive result because it confirmed within 30-50\% uncertainty the energy threshold assumed for EUSO-SPB in simulations.

\section{Trigger performance on flight}

On April 25th, EUSO-SPB was lifted by a Super Pressure Balloon of NASA and flew for about 12 days and operated during all nights without moon. Hundred thousands of packets of data were recorded. About $75 \%$ of them was downloaded before the flight terminated. Data were acquired in nominal runs of 120 seconds. Every hour a much shorter run (40 seconds) was started and data immediately downloaded to understand the general performance of the detector. Fig. 1 shows the overall trigger rate during the entire flight. An indication of the trigger
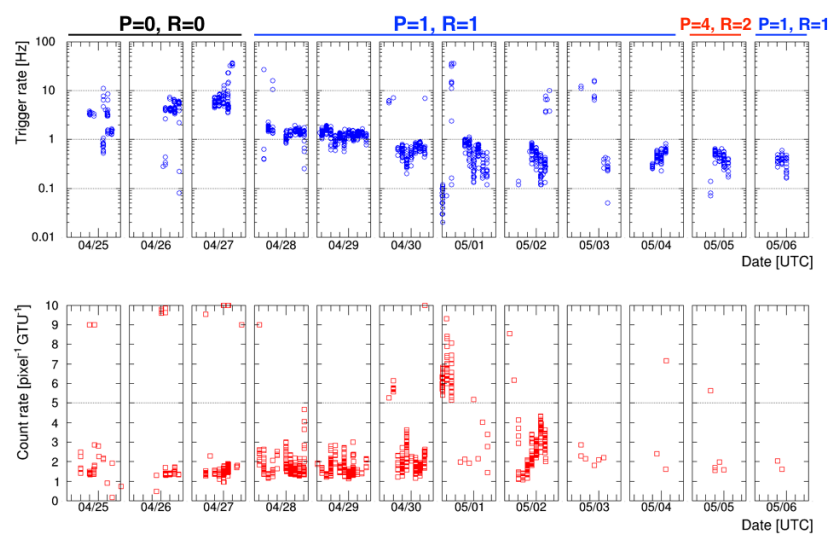

Figure 1: Top: EUSO-SPB trigger rate in flight. Bottom: average count rate measured at pixel level.

modes as a function of time is reported. For the first three nights EUSO-SPB operated in the $P=0$ and $R=0$ mode, which means single GTU trigger. In normal condition the trigger rate was around $\sim 1 \mathrm{~Hz} / \mathrm{EC}$, however, this was too high for the downlink telemetry requirements. During all the rest of the flight, except for one night, the $P=1$ and $R=1$ mode, as used in Utah, was operated. In this case the overall trigger rate was below $2 \mathrm{~Hz} / \mathrm{PDM}$. The peaks above $10 \mathrm{~Hz}$ during the flight are often due to electronics disturbances on one specific EC in some cases related to interference with other add-on units. This situation didn't occur during the Utah tests. The night of May 5th, the $P=4$ and $R=2$ mode (JEM-EUSO trigger mode) was tested. The trigger rate was always below $2 \mathrm{~Hz} / \mathrm{PDM}$. In general, the FLT triggered within the JEM-EUSO requirements (below 7 $\mathrm{Hz} / \mathrm{PDM}$ ). A certain number of events compatible with cosmic rays directly impacting in the detector have been recognized. Simulations are planned to understand and cross-check the rate. The analysis to search for EAS candidates is in progress. Simulations indicate that the number of expected events is of the order of 1 or less in the available data sample.

\section{Acknowledgments}

This work was partially supported by the Italian Ministry of Foreign Affairs and International Cooperation, by the Italian Space Agency through the ASI INFN agreement n. 2017-8-H.0, by NASA award 11-APRA-0058, by the Deutsches Zentrum für Luft- und Raumfahrt, and by the French space agency CNES.

\section{References}

[1] L. Wiencke et al. (JEM-EUSO Coll.): EUSO-SPB1 Mission and Science, PoS(ICRC2017)1097, (2017).

[2] G. Abdellaoui et al. (JEM-EUSO Coll.): Cosmic ray oriented performance studies for the JEM-EUSO first level trigger, Nucl. Instrum. Meth. A866 (2017) 150-163.

[3] J. Bayer et al. (JEM-EUSO Coll.), Second level trigger and Cluster Control Board for the JEM-EUSO mission, Proc. 33th ICRC, \#0432 (2013); arXiv: 1307.7071.

[4] J.H. Adams et al. (JEM-EUSO Coll.): Ground-based tests of JEM-EUSO components at the Telescope Array site, "EUSO-TA, Exp. Astronomy 40 (2015) 301-314. 